\title{
Avaliação da Conformidade de Rótulos de Pescado Comercializado em Caxias do Sul
}

\author{
Conformity Evaluation of Fish Labels Sold in Caxias do Sul
}

\author{
Nicole da Silvaa; Márcia Keller Alves*bc \\ ${ }^{a}$ Faculdade Nossa Senhora de Fátima. RS, Brasil. \\ bUniversidade de Caxias do Sul, Programa de Pós-Graduação Stricto Sensu em Biotecnologia, RS, Brasil. \\ 'Faculdade Nossa Senhora de Fátima, Curso de Tecnologia em Alimentos, RS, Brasil. \\ *E-mail: marcia_nutri@hotmail.com
}

\begin{abstract}
Resumo
A rotulagem dos alimentos é importante, pois é a comunicação entre consumidor e produto. Quando envolvem alimentos de origem animal, principalmente, os altamente perecíveis como os pescados, tal rotulagem deve ser ainda mais criteriosa, garantindo as informações mínimas das condições sanitárias desses alimentos. O objetivo do trabalho foi avaliar a conformidade de rótulos de pescado comercializado na cidade de Caxias do Sul, Rio Grande do Sul. Foi realizado um estudo descritivo, com rótulos de pescado selecionados aleatoriamente, a partir da aplicação de um checklist composto pelas seguintes variáveis: identificação do produto, segurança sanitária, informação nutricional, ingredientes; qualidade técnica do rótulo, qualidade de informação e de informação sobre atendimento ao consumidor. A coleta de dados foi realizada em uma rede de hipermercados. Os dados foram analisados de forma descritiva e os resultados apresentados na forma de frequências relativa e absoluta. Foram analisados 124 rótulos, sendo 74 de filés de peixe congelados e 50 de pescado congelados diversos. Referente à qualidade da rotulagem, as categorias que apresentaram inconformidade com a legislação foram armazenamento e conservação ( $2,72 \%)$, preparo e instrução de uso $(2,72 \%)$ e ingredientes $(2,72 \%)$. Referente à qualidade técnica do rótulo, estava em inconformidade a aderência (14\%), a legibilidade $(19,36 \%)$, a visibilidade $(27,36 \%)$, a ligação gratuita (45,31\%), a ligação paga (45,31\%), a caixa postal (45,31\%) e o e-mail (13,36\%). A maioria dos rótulos de pescados está em conformidade com a legislação vigente, apresentando qualidade sanitária adequada. As categorias que apresentaram maior percentual de inconformidade são categorias técnicas e, portanto, não oferecem risco à saúde do consumidor.
\end{abstract}

Palavras-chave: Embalagem. Pescados. Rotulagem. Vigilância Sanitária.

\begin{abstract}
Food labeling is important because it is the communication between the consumer and the product. When animal origin foodstuffs are involved, particularly highly perishable ones such as fish, such labeling should be even more careful, with minimum information on the health conditions of such foods. The objective was to evaluate the fish labels sold conformity in the city of Caxias do Sul, Rio Grande do Sul, Brazil. A descriptive study was carried out, with randomly selected fish labels, from the application of a checklist composed of the following variables: product identification, health safety, nutritional information; ingredients; the label technical quality, quality of information and information on customer service. Data collection was performed in a network of hypermarkets. The data were analyzed descriptively and the results presented in the form of relative and absolute frequencies. A total of 124 labels were analyzed, of which 74 were from frozen fish fillets and 50 frozen other types of fish. Regarding the labels quality, the categories that presented nonconformity with the legislation were storage and conservation (2.72\%), preparation and instruction of use (2.72\%) and ingredients $(2.72 \%)$. Regarding the label technical quality, adhesion (14\%), readability (19.36\%), visibility (27.36\%), free connection (45.31\%), mailbox (45.31\%) and e-mail (13.36\%). Most fish labels are in compliance with the current legislation, presenting adequate sanitary quality. The categories that presented the highest percentage of nonconformity are technical categories and, therefore, do not pose risk to the consumer health.
\end{abstract}

Keywords: Fish. Labeling. Packaging. Sanitary Surveillance.

\section{Introdução}

No Brasil, a Resolução da Diretora Colegiada (RDC) 259 (ANVISA) aprova o Regulamento Técnico sobre Rotulagem de Alimentos Embalados e conceitua como rotulagem toda inscrição, legenda, imagem ou toda matéria descritiva ou gráfica, escrita, impressa, estampada, gravada, gravada em relevo ou litografada ou colada sobre a embalagem do alimento. As informações obrigatórias na rotulagem são: denominação de venda, lista de ingredientes, conteúdos líquidos, identificação de origem, nome ou razão social do importador (se for o caso), identificação do lote, prazo de validade, instruções sobre preparo e uso do alimento, quando necessário (BRASIL, 2002).

Ainda, segundo a legislação referente à rotulagem de alimentos de origem animal, os rótulos devem apresentar carimbo oficial da Inspeção Federal, categoria do estabelecimento, CNPJ, modo de conservação do produto, marca comercial do produto, data de fabricação, composição do produto e registro no Ministério da Agricultura (BRASIL, 2005).

Entre os produtos de origem animal de importância na alimentação da população está o pescado. O pescado está entre os produtos que deverão apresentar maior demanda global de consumo, juntamente com laticínios e carne, sendo 
o crescimento modesto para peixes e acelerado para frutos do mar (REGO, 2014). Entende-se como pescado todos os peixes, crustáceos, moluscos, quelônios, mamíferos de água doce ou salgada, que sejam utilizados para consumo humano (BRASIL, 1997; BRASIL, 2017).

A produção de pescado, em 2016, segundo a Organização das Nações Unidas para a Agricultura e Alimentação (FAO), foi de 171 milhões de toneladas, das quais $88 \%$ foram utilizadas para o consumo humano direto. O consumo anual de pescado, neste mesmo ano, atingiu em 20,3 kg per capita (FAO, 2018). No Brasil, o consumo de peixes é em média de 9 $\mathrm{kg}$ por habitante por ano, abaixo do consumo mundial, mesmo o país sendo um grande produtor (LOPES; OLIVEIRA; RAMOS, 2016).

O conhecimento sobre o pescado é de extrema importância para que o produto continue sendo introduzido na alimentação (BETANCURT et al., 2016). Todo consumidor tem direito a informações adequadas sobre qualquer produto que esteja adquirindo (BRASIL, 1990) e, em se tratando de alimentos, é de extrema importância que os rótulos estejam de acordo com a legislação vigente, específica para cada tipo de produto (MARZAROTTO; ALVES, 2016). Assim, o presente estudo teve como objetivo avaliar a conformidade de rótulos de pescado comercializado em um estabelecimento situado na cidade de Caxias do Sul, Rio Grande do Sul.

\section{Material e Métodos}

Tratou-se de um estudo descritivo, no qual foram avaliados rótulos selecionados aleatoriamente de pescados, em uma rede de hipermercados da cidade de Caxias do Sul, na região Nordeste do Rio Grande do Sul, no período de março a maio de 2018.

Foram analisados 124 rótulos de pescado, utilizando como referência o checklist elaborado por Bastos et al. (2008). Foram incluídos no presente estudo, os rótulos dos seguintes pescados: filés, como salmão, panga, merluza, linguado, tilápia, outro (quando não especificado), camarão, bacalhau (posta e lobo), moluscos, cação (posta) e salmão (posta).

Foram coletadas dos rótulos as seguintes informações: 1) Identificação do produto: tipo de alimento, identificação do produtor, lote, inscrição "indústria brasileira" e registro; 2) Segurança sanitária: prazo de validade, data de fabricação, carimbo de Serviço de Inspeção Federal (SIF) ou Estadual (SIE); armazenamento e conservação, preparo e instrução de uso (quando necessário); 3) Informações nutricionais; 4) ingredientes (se pertinente); 4) Qualidade técnica do rótulo: aderência, visibilidade (informação aparente, manifesta, perceptível ou que se pode ver), legibilidade (informação nítida, sem manchas ou borrões); 5) Qualidade de informação: coerência e clareza em relação às recomendações técnicas (informação inteligível, compreensível ao entendimento do consumidor); 6) Informação sobre o atendimento ao consumidor: ligação gratuita, ligação paga, caixa postal, e-mail.

Após a coleta de dados, os mesmos foram analisados de forma descritiva, estimando-se frequências das variáveis do estudo por tipo de alimento.

\section{Resultados e Discussão}

Todo consumidor tem direito de saber sobre o produto, a composição, a quantidade, a origem, como consumir, como conservar, o prazo de validade e a informação nutricional do alimento (BRASIL, 1990). Assim, é de extrema importância que os rótulos estejam de acordo com a legislação vigente, específica para cada alimento, principalmente, os de origem animal, que podem apresentar diversos riscos para a saúde humana (MARZAROTTO; ALVES, 2016).

Os rótulos analisados no estudo foram de diversos tipos de pescado encontrados em uma rede de supermercados, totalizando 124 rótulos. Os Quadros 1 e 2 apresentam os resultados encontrados. O Quadro 1 apresenta os dados referentes a rótulos de filé de peixe, que é o produto obtido através de corte único longitudinal da porção muscular desde a parte imediatamente posterior da cabeça até o pedúnculo caudal, no sentido paralelo à coluna vertebral. Por sua vez, o Quadro 2 apresenta os dados referentes a rótulos de outros tipos de pescado congelado, tendo como um dos tipos analisados o lombo, que é a porção dorsal do filé, removido o pedúnculo caudal (BRASIL, 2017).

Quadro 1 - Análise da conformidade de rótulos de filés de peixe congelado por categorias de análise representadas em porcentagem

\begin{tabular}{|c|c|c|c|c|c|c|c|}
\hline \multirow[b]{2}{*}{ Categoria de Análise } & \multicolumn{6}{|c|}{ Tipo de Peixe (filés) } & \multirow[b]{2}{*}{$\begin{array}{c}\text { Total } \\
(\mathrm{n}=74) \\
\% \mathrm{C}\end{array}$} \\
\hline & $\begin{array}{c}\text { Salmão } \\
(n=17) \\
\% C\end{array}$ & $\begin{array}{c}\text { Panga } \\
(n=27) \\
\% \text { C }\end{array}$ & $\begin{array}{c}\text { Merluza } \\
(n=12) \\
\% \mathrm{C}\end{array}$ & $\begin{array}{c}\text { Tilápia } \\
(n=3) \\
\% \mathrm{C}\end{array}$ & $\begin{array}{c}\text { Linguado } \\
(\mathbf{n}=5) \\
\% \mathrm{C}\end{array}$ & $\begin{array}{c}\begin{array}{c}\text { Outro } \\
(n=10)\end{array} \\
\% \text { C }\end{array}$ & \\
\hline \multicolumn{8}{|l|}{ Identificação do produto } \\
\hline Tipo de alimento & 100 & 100 & 100 & 100 & 100 & 100 & 100 \\
\hline Identificação do Produtor & 100 & 100 & 100 & 100 & 100 & 100 & 100 \\
\hline Lote & 100 & 100 & 100 & 100 & 100 & 100 & 100 \\
\hline Inscrição "indústria brasileira" & 100 & 100 & 100 & 100 & 100 & 100 & 100 \\
\hline Registro & 100 & 100 & 100 & 100 & 100 & 100 & 100 \\
\hline \multicolumn{8}{|l|}{ Segurança Sanitária } \\
\hline Prazo de validade & 100 & 100 & 100 & 100 & 100 & 100 & 100 \\
\hline Data de Fabricação & 100 & 100 & 100 & 100 & 100 & 100 & 100 \\
\hline
\end{tabular}




\begin{tabular}{|l|c|c|c|c|c|c|c|}
\hline Carimbo do SIF ou SIE & 100 & 100 & 100 & 100 & 100 & 100 & 100 \\
\hline Armazenamento e conservação & 100 & 100 & 100 & 100 & 100 & 80 & 97,28 \\
\hline Preparo e instrução de uso & 100 & 100 & 100 & 100 & 100 & 80 & 97,28 \\
\hline Informações nutricionais & 100 & 100 & 100 & 100 & 100 & 100 & 100 \\
\hline Ingredientes & 100 & 100 & 100 & 100 & 100 & 80 & 97,28 \\
\hline Qualidade técnica do rotulo & & & & & & & \\
\hline Aderência & 100 & 100 & 100 & 100 & 100 & 100 & 100 \\
\hline Legibilidade & 94,11 & 100 & 100 & 100 & 100 & 100 & 98,64 \\
\hline Visibilidade & 94,11 & 100 & 100 & 100 & 100 & 100 & 98,64 \\
\hline Qualidade da informação & & & & & & & \\
\hline Coerência & 100 & 100 & 100 & 100 & 100 & 100 & 100 \\
\hline $\begin{array}{l}\text { Informações Atendimento ao } \\
\text { Consumidor }\end{array}$ & & & & & & & \\
\hline Ligação gratuita & 100 & 96,29 & 100 & 100 & 100 & 70 & 94,59 \\
\hline Ligação paga & 100 & 22,2 & 100 & 100 & 100 & 70 & 94,59 \\
\hline Caixa postal & 100 & 22,2 & 100 & 100 & 100 & 70 & 94,59 \\
\hline E-mail & 100 & 96,29 & 100 & 100 & 100 & 100 & 98,64 \\
\hline Clareza nas informações & 100 & 100 & 100 & 100 & 100 & 100 & 100 \\
\hline
\end{tabular}

$\% \mathrm{C}=$ percentual de conformidade.

Fonte: Dados da pesquisa.

Quadro 2 - Análise da conformidade de rótulos de pescado congelado por categorias de análise

\begin{tabular}{|c|c|c|c|c|c|c|c|}
\hline \multirow[b]{2}{*}{ Categoria de Análise } & \multicolumn{6}{|c|}{ Tipo de Alimento } & \multirow[b]{2}{*}{$\begin{array}{c}\text { Total } \\
(\mathrm{n}=50) \\
\% \mathrm{C}\end{array}$} \\
\hline & $\begin{array}{c}\text { Camarão } \\
(n=17) \\
\% \mathrm{C}\end{array}$ & $\begin{array}{c}\text { Bacalhau } \\
\text { Posta } \\
(n=9) \% \mathrm{C}\end{array}$ & $\begin{array}{c}\text { Bacalhau } \\
\text { Lombo } \\
(n=4) \% C\end{array}$ & $\begin{array}{c}\text { Moluscos } \\
(n=8) \\
\% \text { C }\end{array}$ & $\begin{array}{l}\text { Cação } \\
\text { Posta } \\
(n=5) \\
\% \text { C }\end{array}$ & $\begin{array}{c}\text { Salmão } \\
(n=7) \\
\% \text { C }\end{array}$ & \\
\hline \multicolumn{8}{|l|}{ Identificação do produto } \\
\hline Tipo de alimento & 100 & 100 & 100 & 100 & 100 & 100 & 100 \\
\hline Identificação do Produtor & 100 & 100 & 100 & 100 & 100 & 100 & 100 \\
\hline Lote & 100 & 100 & 100 & 100 & 100 & 100 & 100 \\
\hline Inscrição "indústria brasileira" & 100 & 100 & 100 & 100 & 100 & 100 & 100 \\
\hline Registro & 100 & 100 & 100 & 100 & 100 & 100 & 100 \\
\hline \multicolumn{8}{|l|}{ Segurança Sanitária } \\
\hline Prazo de validade & 100 & 100 & 100 & 100 & 100 & 100 & 100 \\
\hline Data de Fabricação & 100 & 100 & 100 & 100 & 100 & 100 & 100 \\
\hline Carimbo do SIF ou SIE & 100 & 100 & 100 & 100 & 100 & 100 & 100 \\
\hline Armazenamento e conservação & 100 & 100 & 100 & 100 & 100 & 100 & 100 \\
\hline Preparo e instrução de uso & 100 & 100 & 100 & 100 & 100 & 100 & 100 \\
\hline Informações nutricionais & 100 & 100 & 100 & 100 & 100 & 100 & 100 \\
\hline Ingredientes & 100 & 100 & 100 & 100 & 100 & 100 & 100 \\
\hline \multicolumn{8}{|l|}{ Qualidade técnica do rotulo } \\
\hline Aderência & 94,11 & 100 & 100 & 100 & 100 & 100 & 86 \\
\hline Legibilidade & 94,11 & 100 & 100 & 100 & 100 & 71,42 & 82 \\
\hline Visibilidade & 94,11 & 100 & 100 & 100 & 100 & 14,2 & 74 \\
\hline \multicolumn{8}{|l|}{ Qualidade da informação } \\
\hline Coerência & 100 & 100 & 100 & 100 & 100 & 100 & 100 \\
\hline \multicolumn{8}{|l|}{$\begin{array}{l}\text { Informações atendimento ao } \\
\text { consumidor }\end{array}$} \\
\hline Ligação gratuita & 64,70 & 100 & 100 & 0 & 100 & 100 & 60 \\
\hline Ligação paga & 64,70 & 100 & 100 & 0 & 100 & 100 & 60 \\
\hline Caixa postal & 64,70 & 100 & 100 & 0 & 100 & 100 & 60 \\
\hline E-mail & 64,70 & 100 & 100 & 0 & 100 & 100 & 60 \\
\hline Clareza nas informações & 100 & 100 & 100 & 100 & 100 & 100 & 100 \\
\hline
\end{tabular}

$\% \mathrm{C}=$ percentual de conformidade.

Fonte: Dados da pesquisa.

Foi encontrado que $97,28 \%$ da rotulagem está em conformidade nos filés de peixes congelados. As informações contidas em rótulos de pescado referentes aos aspectos sanitários, especialmente, as datas de fabricação e de validade, selos de inspeção e modo de conservação do produto, devem instruir o consumidor à manipulação deste produto, bem como 
seu armazenamento e consumo no prazo adequado, de modo a prevenir problemas para a saúde humana, causado pelos alimentos, também conhecidos por doenças transmitidas por alimentos (DTA).

Outro item com pequeno percentual de inconformidade foi o Preparo e Instrução de uso para filés de peixes, o que pode trazer inúmeras dificuldades para o consumidor, especialmente, no que diz respeito ao descongelamento correto do produto. A informação sobre a temperatura do produto para conservação em ambiente doméstico é extremamente importante, pois o descongelamento incorreto do pescado aumenta a quantidade de água livre no músculo do peixe, o que pode gerar multiplicação indesejada de micro-organismos deteriorantes (SANTOS, 2017). A legislação preconiza que sempre que necessário, o rótulo do alimento contenha as instruções sobre o modo apropriado de uso, incluindo a reconstituição, o descongelamento ou o tratamento certo que deve ser dado (BRASIL, 2002).

Referente ao Armazenamento e Conservação, embora o percentual de inconformidade tenha sido baixo, devese dar muita atenção a este item, uma vez que alimentos congelados exigem temperaturas e formas de armazenamento específicas, especialmente o pescado, por apresentar grande propensão ao deterioramento. A RDC 259 declara como obrigatória a apresentação nos rótulos de alimentos o item de armazenamento e conservação do alimento, com uma legenda legível, além de sua temperatura adequada e o tempo de durabilidade que o produtor garante (BRASIL, 2002). O pescado deve ser mantido em uma temperatura não superior a $-18{ }^{\circ} \mathrm{C}$ para ser considerado adequado para consumo (BRASIL, 2017).

A categoria Ingredientes informa o que está presente na preparação do alimento, assim evitando que indivíduos intolerantes ou alérgicos consumam. Nos rótulos do pescado de todas as espécies é obrigatório que apareça a lista de ingredientes, mostrando se contém ingrediente de outro alimento (BRASIL, 2002). A RDC 26 da ANVISA assegura a informação da presença de ingredientes em produtos, incluindo a presença de elementos ou traço, resultantes da contaminação durante a produção (BRASIL, 2015).

Em todos os rótulos, a categoria Tipo de Produto estava $100 \%$ em conformidade. Tão importante quanto estar correta a denominação do produto, é o consumidor encontrar dentro da embalagem o produto que está comprando. Um exemplo deste tipo de fraude ocorre com o bacalhau, em que espécies de peixes salgados e secos são comercializados como sendo o próprio bacalhau (AGUIAR, 2015). Por isso, em 2015, o MAPA iniciou o programa de controle oficial da fraude por substituição de espécie em pescado, tomando como base a

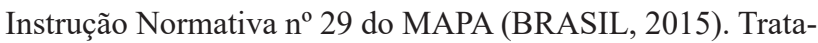
se de uma ferramenta que esclarece à indústria as regras de denominação de nomes populares do pescado, sem deixar espaço para dúvidas para o consumidor e combater a fraude em pescado.
Referente à qualidade técnica do rótulo, tanto em rótulos de filé de peixe quanto em rótulos dos pescados analisados, foram encontroadas inconformidades. Desse modo, o consumidor pode não estar recebendo todas as informações sobre o produto de forma adequada. A rotulagem é toda a identidade do produto, sendo a ligação do produtor com o consumidor, mostrando tudo o que deve ser conhecido sobre o alimento (MARZAROTTO; ALVES, 2016).

A Constituição Federal garante, através da RDC 259 (BRASIL, 2002), que as informações fornecidas nas embalagens de produtos devem ser claras, de fácil entendimento e completas para o consumidor. Os consumidores, cada vez mais preocupados com o que estão adquirindo para o consumo, acabam criando o hábito da leitura de rótulos dos alimentos e, desse modo, exercendo seus direitos (MARZAROTTO; ALVES, 2016).

A categoria que obteve menor percentual de adequação em ambas as tabelas foi a referente à informação sobre atendimento ao consumidor.

O Serviço de Atendimento ao Consumir (SAC) tem a finalidade de resolver as demandas dos consumidores sobre informações, dúvidas, reclamações, entre outros (BRASIL, 1990) e aproximar o consumidor e o produtor (BASTOS et al., 2008). Entretanto, não há no Código de Defesa do Consumidor ou em outras legislações qualquer obrigatoriedade quanto a este serviço. O SAC pode ocorrer via telefônica sem ônus ao consumidor (a ligação deverá ser gratuita) ou eletrônica (endereço eletrônico e home page) (BRASIL, 2008).

As demais categorias de análise estavam 100\% em conformidade com a legislação, mostrando que o pescado brasileiro garante boa qualidade, no que se refere às informações prestadas através de sua rotulagem. A leitura dos rótulos é não somente um direito dos cidadãos, no que diz respeito às informações contidas, mas também um dever, considerando que toda a identificação do produto está em sua rotulagem. Este hábito de leitura dos rótulos para compra de produto, em especial pescados, deve ser passado de geração para geração e continuamente estimulado. Entretanto, é necessário que os rótulos sejam preenchidos de forma correta, de acordo com a legislação, para garantir a proteção à saúde dos consumidores (MONTEIRO et al., 2007).

Sendo assim, cabe às entidades governamentais não só fiscalizar o cumprimento das leis, mas também orientar os produtores, os comerciantes e os distribuidores de alimentos de modo que se produzam alimentos de qualidade e segurança e, assim, o consumidor possa escolher, de forma adequada, os alimentos que deseja consumir (BARROS et al., 2012).

\section{Conclusão}

A partir dos resultados se encontrou que a maioria dos rótulos de pescados está em conformidade com a legislação atual vigente, apresentando qualidade técnica adequada. Algumas categorias estavam em inconformidade, categorias essas que fornecem informações importantes ao 
consumidor. No entanto, não são categorias que representam inconformidade sanitária do pescado e, portanto, não oferecem risco à saúde do consumidor.

\section{Referências}

AGUIAR, A.B.L. Qualidade do pescado salgado seco nos seus aspectos físico-químicos e de rotulagem. Uberlândia: Universidade Federal de Uberlândia, 2015.

ABIA - Associação Brasileira das Indústrias da Alimentação. Consumo de alimentos em novo patamar. São Paulo: ABIA, 1997.

BARROS, A.C et al. Análise de rotulagem de pescados comercializados em estabelecimentos do município de PetrolinaPE. CONNEPI, 2012.

BASTOS, A. et al. Avaliação da qualidade sanitária dos rótulos de alimentos embalados de origem animal. Rev. Baiana Saúde Pública; v.32, n.2, p.218-231, 2008.

BETANCURT, L.F.; ESPINOSA, H.R.; VALÊNCIA-, Y D. Caracterización del consumo de pescado y mariscos en población universitária de la ciudad de Medellín-Colombia. Rev. Univ. Salud., v.18, n.2, p.257-265, 2016.

BRASIL. Agencia Nacional de Vigilância Sanitária. ANVISA. Resolução da Diretoria Colegiada - RDC n ${ }^{\circ} 26$, de 2 de julho de 2015. Dispõe sobre os requisitos para rotulagem obrigatória dos principais alimentos que causam alergias alimentares. Brasília, Diário Oficial da União de 3 de julho de 2015.

BRASIL. Agência Nacional de Vigilância Sanitária. ANVISA. Resolução da Diretoria Colegiada. RDC no 259, de 20 de setembro de 2002. Regulamento técnico de alimentos embalados. Brasília, Diário Oficial da União de 18 de setembro de 2002. Disponível em: https://lcqa.farmacia.ufg.br/up/912/o/resoluo rdc_n_259_2002_-_rotulagem_em_geral.pdf. Acessado em: $1 \overline{8}$ jun. 2019 .

BRASIL. Ministério da Agricultura, Pecuária e Abastecimento Instrução normativa $\mathrm{n}^{\circ} 21$, de 31 de maio de 2017. Regulamento técnico que fixa a identidade e as características da qualidade que deve apresentar o peixe congelado. Brasília, Diário Oficial da União de 07/06/2017.

BRASIL. Ministério da Agricultura, Pecuária e Abastecimento. MAPA. Instrução normativa ${ }^{\circ} 22$, de 24 de novembro de 2005. Regulamento técnico para rotulagem de produtos de origem animal embalado. Brasília, Diário Oficial da União de 25 de novembro de 2005.
BRASIL. Ministério da Agricultura, Pecuária e Abastecimento. MAPA. Instrução normativa $n^{\circ} 29$, de 23 de setembro de 2015. Determina a padronização de nomes populares para espécies de peixe. Brasília, Diário Oficial da União de 24 de setembro de 2015.

BRASIL. Ministério da Agricultura, Pecuária e Abastecimento. MAPA. Portaria $n^{\circ} 185$, de 13 de maio de 1997. Regulamento técnico de identidade e qualidade de peixe fresco. Brasília, Diário Oficial da União de 19 de maio de 1997.

BRASIL. Presidência da República. Casa Civil. Subchefia para Assuntos Jurídicos. Decreto $\mathrm{n}^{\circ}$ 6.523, de 31 de julho de 2008. Regulamenta a Lei no 8.078, de 11 de setembro de 1990, para fixar normas gerais sobre o Serviço de Atendimento ao Consumidor SAC. Brasília, Diário Oficial da União de $1^{\circ}$ de agosto de 2008.

BRASIL. Presidência da República. Casa Civil. Subchefia para Assuntos Jurídicos. LEI No 8.078, de 11 de setembro de 1990. Dispõe sobre a proteção do consumidor e dá outras providências. Brasília, Diário Oficial da União de 12 de setembro de 1990. Disponível em: http://www.planalto.gov.br/ccivil_03/leis/18078. htm. Acessado em: 18 jun. 2019.

FAO. Organización de las Naciones Unidas para la Alimentación y la Agricultura. El estado mundial de la pesca y la acuicultura 2018. Cumplir los objetivos de desarrollo sostenible. Roma. Licencia: CC BY-NC-SA 3.0 IGO.

LOPES, I.G.; OLIVEIRA, R.G.; RAMOS, F.M. Perfil do consumo de peixes pela população brasileira. Biota Amazônia, v.6, n.2, p.62-65, 2016. doi: 10.18561/2179-5746/biotaamazonia. v6n2p62-65.

MARZAROTTO, B., ALVES, M.K. Leitura de rótulos de alimentos por frequentadores de um estabelecimento comercial. Rev. Eletr. Ciênc. Saúde, v.10, n.2, p.102-108, 2016.

MONTEIRO, J.; PIRES, P.; BARROS, R. Avaliação do grau de conformidade da rotulagem de gêneros alimentícios de origem animal face à legislação nacional e comunitária em vigor. Rev. Port. Ciênc. Vet., v.102, n.563-564, p.343-349, 2007.

REGO, R. A. Conclusões e identificação de desafios e oportunidades. In: Sustentabilidade e Sustentação da Produção de Alimentos no Brasil: Consumo de Alimentos: implicações para a produção agropecuária. Brasília: Centro de Gestão e Estudos Estratégicos, 2014.

SANTOS, R.C. Rótulos de alimentos refrigerados e manipulados em supermercados. Brasília: UnB, 2017. 